\title{
A unified proof of several inequalities and some new inequalities involving Neuman-Sándor mean
}

$$
\text { Feng Qi and Wen-Hui Li }
$$




\title{
A UNIFIED PROOF OF SEVERAL INEQUALITIES AND SOME NEW INEQUALITIES INVOLVING NEUMAN-SÁNDOR MEAN
}

\author{
FENG QI AND WEN-HUI LI
}

Received 21 March, 2014

\begin{abstract}
In the paper, by finding linear relations of differences between some means, the authors supply a unified proof of several double inequalities for bounding Neuman-Sándor means in terms of the arithmetic, harmonic, and contra-harmonic means and discover some new sharp inequalities involving Neuman-Sándor, contra-harmonic, root-square, and other means of two positive real numbers.
\end{abstract}

2010 Mathematics Subject Classification: 26E60; 26D07; 41A30

Keywords: mean, inequality, Neuman-Sándor mean, monotonicity, unified proof, hyperbolic sine, hyperbolic cosine

\section{INTRODUCTION}

It is well known that the quantities

$$
\begin{aligned}
A(a, b) & =\frac{a+b}{2}, & G(a, b) & =\sqrt{a b}, \\
H(a, b) & =\frac{2 a b}{a+b}, & \bar{C}(a, b) & =\frac{2\left(a^{2}+a b+b^{2}\right)}{3(a+b)}, \\
C(a, b) & =\frac{a^{2}+b^{2}}{a+b}, & P(a, b) & =\frac{a-b}{4 \arctan \sqrt{a / b}-\pi}, \\
Q(a, b) & =\sqrt{\frac{a^{2}+b^{2}}{2},} & T(a, b) & =\frac{a-b}{2 \arctan \frac{a-b}{a+b}}
\end{aligned}
$$

are respectively called in the literature the arithmetic, geometric, harmonic, centroidal, contra-harmonic, first Seiffert, root-square, and second Seiffert means of two positive real numbers $a$ and $b$ with $a \neq b$.

For $a, b>0$ with $a \neq b$, Neuman-Sándor mean $M(a, b)$ is defined in [11] by

$$
M(a, b)=\frac{a-b}{2 \operatorname{arcsinh} \frac{a-b}{a+b}},
$$


where $\operatorname{arcsinh} x=\ln \left(x+\sqrt{x^{2}+1}\right)$ is the inverse hyperbolic sine function. At the same time, a chain of inequalities

$$
G(a, b)<L_{-1}(a, b)<P(a, b)<A(a, b)<M(a, b)<T(a, b)<Q(a, b)
$$

were given in [11], where

$$
L_{p}(a, b)= \begin{cases}{\left[\frac{b^{p+1}-a^{p+1}}{(p+1)(b-a)}\right]^{1 / p},} & p \neq-1,0 \\ \frac{1}{e}\left(\frac{b^{b}}{a^{a}}\right)^{1 /(b-a)} & p=0 \\ \frac{b-a}{\ln b-\ln a}, & p=-1\end{cases}
$$

is the $p$-th generalized logarithmic mean of $a$ and $b$ with $a \neq b$.

In $[11,12]$, it was established that

$$
\begin{gathered}
A(a, b)<M(a, b)<T(a, b), \quad P(a, b)<M(a, b)<T^{2}(a, b), \\
A(a, b) T(a, b)<M^{2}(a, b)<\frac{A^{2}(a, b)+T^{2}(a, b)}{2}
\end{gathered}
$$

for $a, b>0$ with $a \neq b$.

For $0<a, b<\frac{1}{2}$ with $a \neq b$, Ky Fan type inequalities

$$
\begin{aligned}
\frac{G(a, b)}{G(1-a, 1-b)}<\frac{L_{-1}(a, b)}{L_{-1}(1-a, 1-b)}<\frac{P(a, b)}{P(1-a, 1-b)} \\
\quad<\frac{A(a, b)}{A(1-a, 1-b)}<\frac{M(a, b)}{M(1-a, 1-b)}<\frac{T(a, b)}{T(1-a, 1-b)}
\end{aligned}
$$

were presented in [11, Proposition 2.2].

In [8], it was showed that the double inequality

$$
L_{p_{0}}(a, b)<M(a, b)<L_{2}(a, b)
$$

holds for all $a, b>0$ with $a \neq b$ and for $p_{0}=1.843 \ldots$, where $p_{0}$ is the unique solution of the equation $(p+1)^{1 / p}=2 \ln (1+\sqrt{2})$.

In [10], Neuman proved that the double inequalities

$$
\alpha Q(a, b)+(1-\alpha) A(a, b)<M(a, b)<\beta Q(a, b)+(1-\beta) A(a, b)
$$

and

$$
\lambda C(a, b)+(1-\lambda) A(a, b)<M(a, b)<\mu C(a, b)+(1-\mu) A(a, b)
$$

hold for all $a, b>0$ with $a \neq b$ if and only if

$$
\alpha \leq \frac{1-\ln (1+\sqrt{2})}{(\sqrt{2}-1) \ln (1+\sqrt{2})}=0.3249 \ldots, \quad \beta \geq \frac{1}{3}
$$


and

$$
\lambda \leq \frac{1-\ln (1+\sqrt{2})}{\ln (1+\sqrt{2})}=0.1345 \ldots, \quad \mu \geq \frac{1}{6} .
$$

In [20, Theorems 1.1 to 1.3], it was found that the double inequalities

$$
\begin{gathered}
\alpha_{1} H(a, b)+\left(1-\alpha_{1}\right) Q(a, b)<M(a, b)<\beta_{1} H(a, b)+\left(1-\beta_{1}\right) Q(a, b), \\
\alpha_{2} G(a, b)+\left(1-\alpha_{2}\right) Q(a, b)<M(a, b)<\beta_{2} G(a, b)+\left(1-\beta_{2}\right) Q(a, b),
\end{gathered}
$$

and

$$
\alpha_{3} H(a, b)+\left(1-\alpha_{3}\right) C(a, b)<M(a, b)<\beta_{3} H(a, b)+\left(1-\beta_{3}\right) C(a, b)
$$

hold for all $a, b>0$ with $a \neq b$ if and only if

$$
\begin{gathered}
\alpha_{1} \geq \frac{2}{9}=0.2222 \ldots, \quad \beta_{1} \leq 1-\frac{1}{\sqrt{2} \ln (1+\sqrt{2})}=0.1977 \ldots, \\
\alpha_{2} \geq \frac{1}{3}=0.3333 \ldots, \quad \beta_{2} \leq 1-\frac{1}{\sqrt{2} \ln (1+\sqrt{2})}=0.1977 \ldots, \\
\alpha_{3} \geq 1-\frac{1}{2 \ln (1+\sqrt{2})}=0.4327 \ldots, \quad \beta_{3} \leq \frac{5}{12}=0.4166 \ldots
\end{gathered}
$$

In [19, Theorem 3.1], it was established that the double inequality

$$
\alpha I(a, b)+(1-\alpha) Q(a, b)<M(a, b)<\beta I(a, b)+(1-\beta) Q(a, b)
$$

holds for all $a, b>0$ with $a \neq b$ if and only if

$$
\alpha \geq \frac{1}{2} \quad \text { and } \quad \beta \leq \frac{e[\sqrt{2} \ln (1+\sqrt{2})-1]}{(\sqrt{2} e-2) \ln (1+\sqrt{2})}=0.4121 \ldots
$$

For more information on this topic, please refer to $[1-3,5,7-10,12-14,16-18,20]$ and plenty of references cited therein.

The first goal of this paper is, by finding linear relations of differences between some means, to supply a unified proof of inequalities (1.1) and (1.2).

The second purpose of this paper is to establish some new sharp inequalities involving Neuman-Sándor, centroidal, contra-harmonic, and root-square means of two positive real numbers $a$ and $b$ with $a \neq b$.

\section{LEMMAS}

In order to attain our aims, the following lemmas are needed.

Lemma 1 ([15, Lemma 1.1]). Suppose that the power series $f(x)=\sum_{n=0}^{\infty} a_{n} x^{n}$ and $g(x)=\sum_{n=0}^{\infty} b_{n} x^{n}$ have the radius of convergence $r>0$ and $b_{n}>0$ for all $n \in$ $\mathbb{N}=\{0,1,2, \ldots\}$. Let $h(x)=\frac{f(x)}{g(x)}$. Then the following statements are true. 
(1) If the sequence $\left\{\frac{a_{n}}{b_{n}}\right\}_{n=0}^{\infty}$ is (strictly) increasing (decreasing), then $h(x)$ is also (strictly) increasing (decreasing) on $(0, r)$.

(2) If the sequence $\left\{\frac{a_{n}}{b_{n}}\right\}$ is (strictly) increasing (decreasing) for $0<n \leq n_{0}$ and (strictly) decreasing (increasing) for $n>n_{0}$, then there exists $x_{0} \in(0, r)$ such that $h(x)$ is (strictly) increasing (decreasing) on $\left(0, x_{0}\right)$ and (strictly) decreasing (increasing) on $\left(x_{0}, r\right)$.

Lemma 2. Let

$$
h_{1}(x)=\frac{\sinh x-x}{2 x \sinh ^{2} x} .
$$

Then $h_{1}(x)$ is strictly decreasing on $(0, \infty)$ and has the limit $\lim _{x \rightarrow 0^{+}} h_{1}(x)=\frac{1}{12}$.

Proof. Let $f_{1}(x)=\sinh x-x$ and $f_{2}(x)=2 x \sinh ^{2} x=x \cosh 2 x-x$. Using the power series

$$
\sinh x=\sum_{n=0}^{\infty} \frac{x^{2 n+1}}{(2 n+1) !} \quad \text { and } \quad \cosh x=\sum_{n=0}^{\infty} \frac{x^{2 n}}{(2 n) !},
$$

we can express the functions $f_{1}(x)$ and $f_{2}(x)$ as

$$
f_{1}(x)=\sum_{n=0}^{\infty} \frac{x^{2 n+3}}{(2 n+3) !} \quad \text { and } \quad f_{2}(x)=\sum_{n=0}^{\infty} \frac{2^{2 n+2} x^{2 n+3}}{(2 n+2) !}
$$

Hence, we have

$$
h_{1}(x)=\frac{\sum_{n=0}^{\infty} a_{n} x^{2 n}}{\sum_{n=0}^{\infty} b_{n} x^{2 n}}
$$

where $a_{n}=\frac{1}{(2 n+3) !}$ and $b_{n}=\frac{2^{2 n+2}}{(2 n+2) !}$. Let $c_{n}=\frac{a_{n}}{b_{n}}$. Then $c_{n}=\frac{1}{(2 n+3) 2^{2 n+2}}$ and

$$
c_{n+1}-c_{n}=\frac{-(6 n+17)}{(2 n+3)(2 n+5) 2^{2 n+4}}<0 .
$$

As a result, by Lemma 1, it follows that the function $h_{1}(x)$ is strictly decreasing on $(0, \infty)$.

From (2.4), it is easy to see that $\lim _{x \rightarrow 0^{+}} h_{1}(x)=\frac{a_{0}}{b_{0}}=\frac{1}{12}$. The proof of Lemma 2 is complete.

Lemma 3. Let

$$
h_{2}(x)=\frac{1-\frac{\sinh x}{x}+\frac{\sinh ^{2} x}{3}}{\cosh x-\frac{\sinh x}{x}} .
$$

Then $h_{2}(x)$ is strictly increasing on $(0, \infty)$ and has the limit $\lim _{x \rightarrow 0^{+}} h_{2}(x)=\frac{1}{2}$. 
Proof. Let

$$
f_{3}(x)=1-\frac{\sinh x}{x}+\frac{\sinh ^{2} x}{3}=1-\frac{\sinh x}{x}+\frac{\cosh 2 x-1}{6}
$$

and

$$
f_{4}(x)=\cosh x-\frac{\sinh x}{x} .
$$

Making use of the power series in (2.2) shows that

$$
f_{3}(x)=\sum_{n=0}^{\infty} \frac{(2 n+3) 2^{2 n+2}-6}{6(2 n+3) !} x^{2 n+2} \text { and } \quad f_{4}(x)=\sum_{n=0}^{\infty} \frac{2 n+2}{(2 n+3) !} x^{2 n+2} \text {. }
$$

Therefore, we have

$$
h_{2}(x)=\frac{\sum_{n=0}^{\infty} a_{n} x^{2 n+2}}{\sum_{n=0}^{\infty} b_{n} x^{2 n+2}},
$$

where $a_{n}=\frac{(2 n+3) 2^{2 n+2}-6}{6(2 n+3) !}$ and $b_{n}=\frac{2 n+2}{(2 n+3) !}$. Let $c_{n}=\frac{a_{n}}{b_{n}}$. Then

$$
c_{n}=\frac{(2 n+3) 2^{2 n+1}-3}{6(n+1)}
$$

and

$$
c_{n+1}-c_{n}=\frac{3+7 \cdot 2^{2 n+2}+21 n \cdot 2^{2 n+1}+3 n^{2} \cdot 2^{2 n+2}}{6(n+1)(n+2)}>0 .
$$

Accordingly, by Lemma 1 , it follows that the function $h_{2}(x)$ is strictly increasing on $(0, \infty)$.

It is clear that $\lim _{x \rightarrow 0^{+}} h_{2}(x)=\frac{a_{0}}{b_{0}}=\frac{1}{2}$. The proof of Lemma 3 is complete.

Lemma 4. Let

$$
h_{3}(x)=\frac{\cosh x-\frac{\sinh x}{x}}{1+\sinh ^{2} x-\frac{\sinh x}{x}} .
$$

Then $h_{3}(x)$ is strictly decreasing on $(0, \infty)$ and has the limit $\lim _{x \rightarrow 0^{+}} h_{3}(x)=\frac{2}{5}$.

Proof. Let

$$
f_{5}(x)=\cosh x-\frac{\sinh x}{x}
$$

and

$$
f_{6}(x)=1+\sinh ^{2} x-\frac{\sinh x}{x}=1-\frac{\sinh x}{x}+\frac{\cosh 2 x-1}{2} .
$$

Utilizing the power series in (2.2) gives

$$
f_{5}(x)=\sum_{n=0}^{\infty} \frac{2 n+2}{(2 n+3) !} x^{2 n+2} \quad \text { and } \quad f_{6}(x)=\sum_{n=0}^{\infty} \frac{(2 n+3) 2^{2 n+1}-1}{(2 n+3) !} x^{2 n+2} .
$$


This implies that

$$
h_{3}(x)=\frac{\sum_{n=0}^{\infty} a_{n} x^{2 n+2}}{\sum_{n=0}^{\infty} b_{n} x^{2 n+2}},
$$

where $a_{n}=\frac{2 n+2}{(2 n+3) !}$ and $b_{n}=\frac{(2 n+3) 2^{2 n+1}-1}{(2 n+3) !}$. Let $c_{n}=\frac{a_{n}}{b_{n}}$. Then

$$
c_{n}=\frac{2 n+2}{(2 n+3) 2^{2 n+1}-1}
$$

and

$$
c_{n+1}-c_{n}=-\frac{2\left(1+7 \cdot 2^{2 n+2}+21 n \cdot 2^{2 n+1}+3 n^{2} \cdot 2^{2 n+2}\right)}{\left(3 \cdot 2^{2 n+1}+n \cdot 2^{2 n+2}-1\right)\left(5 \cdot 2^{2 n+3}+n \cdot 2^{2 n+4}-1\right)}<0 .
$$

In light of Lemma 1 , we obtain that the function $h_{3}(x)$ is strictly decreasing on $(0, \infty)$.

It is obvious that $\lim _{x \rightarrow 0^{+}} h_{3}(x)=\frac{a_{0}}{b_{0}}=\frac{2}{5}$. The proof of Lemma 4 is complete.

\section{A UNIFIED PROOF OF INEQUALITIES (1.1) AND (1.2)}

Now we are in a position to supply a unified proof of inequalities (1.1) and (1.2) and, as corollaries, to establish some new inequalities involving Neuman-Sándor, contra-harmonic, centroidal, and root-square means of two positive real numbers $a$ and $b$ with $a \neq b$.

It is not difficult to see that the inequalities (1.1) and (1.2) can be rearranged respectively as

$$
\lambda-1<\frac{M(a, b)-C(a, b)}{C(a, b)-A(a, b)}<\mu-1
$$

and

$$
-\alpha_{3}<\frac{M(a, b)-C(a, b)}{C(a, b)-H(a, b)}<-\beta_{3} .
$$

The denominators in (3.1) and (3.2) meet

$$
2[C(a, b)-A(a, b)]=C(a, b)-H(a, b)=\frac{(a-b)^{2}}{a+b}
$$

which were presented in [4, Eq. (4.4)]. This implies that the inequalities (1.1) and (1.2) are identical up to a scalar. Therefore, it is sufficient to prove one of the two inequalities (1.1) and (1.2).

By a direct calculation, we also find

$$
\begin{aligned}
6[\bar{C}(a, b)-A(a, b)]=3 & {[C(a, b)-\bar{C}(a, b)]=2[A(a, b)-H(a, b)] } \\
= & \frac{3}{2}[\bar{C}(a, b)-H(a, b)]=\frac{(a-b)^{2}}{a+b} \triangleq C H(a, b) .
\end{aligned}
$$


So, it is natural to raise a problem: what are the best constants $\alpha$ and $\beta$ such that the double inequality

$$
\alpha<\frac{M(a, b)-C(a, b)}{C H(a, b)}<\beta
$$

holds for all $a, b>0$ with $a \neq b$ ? The following theorem gives a solution to this problem.

Theorem 1. The double inequality (3.5) holds for all $a, b>0$ with $a \neq b$ if and only if

$$
\alpha \leq \frac{1}{2 \ln (1+\sqrt{2})}-1=-0.4327 \ldots \quad \text { and } \quad \beta \geq-\frac{5}{12}=-0.4166 \ldots
$$

Proof. Without loss of generality, we assume that $a>b>0$. Let $x=\frac{a}{b}$. Then $x>1$ and

$$
\frac{M(a, b)-C(a, b)}{C H(a, b)}=\frac{\frac{x-1}{2 \operatorname{arcsinh} \frac{x-1}{x+1}}-\frac{x^{2}+1}{x+1}}{\frac{(x-1)^{2}}{x+1}} .
$$

Let $t=\frac{x-1}{x+1}$. Then $t \in(0,1)$ and

$$
\frac{M(a, b)-C(a, b)}{C H(a, b)}=\frac{\frac{t}{\operatorname{arcsinh} t}-t^{2}-1}{2 t^{2}} .
$$

Let $t=\sinh \theta$ for $\theta \in(0, \ln (1+\sqrt{2}))$. Then

$$
\frac{M(a, b)-C(a, b)}{C H(a, b)}=\frac{\frac{\sinh \theta}{\theta}-\sinh ^{2} \theta-1}{2 \sinh ^{2} \theta}=\frac{\sinh \theta-\theta}{2 \theta \sinh ^{2} \theta}-\frac{1}{2} .
$$

In virtue of Lemma 2, Theorem 1 is thus proved.

Corollary 1. The double inequality

$$
\alpha C H(a, b)+M(a, b)<C(a, b)<\beta C H(a, b)+M(a, b)
$$

holds for all $a, b>0$ with $a \neq b$ if and only if $\alpha \leq \frac{5}{12}=0.4166 \ldots$ and

$$
\beta \geq 1-\frac{1}{2 \ln (1+\sqrt{2})}=0.4327 \ldots
$$

Corollary 2. The double inequality

$$
\alpha C H(a, b)+M(a, b)<\bar{C}(a, b)<\beta C H(a, b)+M(a, b)
$$

holds for all $a, b>0$ with $a \neq b$ if and only if $\alpha \leq \frac{1}{12}=0.0833 \ldots$ and

$$
\beta \geq \frac{2}{3}-\frac{1}{2 \ln (1+\sqrt{2})}=0.0993 \ldots
$$




\section{SOME NEW INEQUALITIES INVOLVING NEUMAN-SÁNDOR MEAN}

Finally we further establish some new inequalities involving Neuman-Sándor, centroidal, root-square, and other means.

Theorem 2. The inequality

$$
M(a, b)>\lambda C H(a, b)
$$

holds for all $a, b>0$ with $a \neq b$ if and only if $\lambda \leq \frac{1}{2 \ln (1+\sqrt{2})}=0.5672 \ldots$

Proof. It is clear that

$$
\frac{M(a, b)}{C H(a, b)}=\frac{(a-b)(a+b)}{(a-b)^{2} 2 \operatorname{arcsinh} \frac{a-b}{a+b}}=\frac{a+b}{a-b} \frac{1}{2 \operatorname{arcsinh} \frac{a-b}{a+b}} .
$$

Without loss of generality, we assume that $a>b>0$. Let $x=\frac{a-b}{a+b}$. Then $x \in(0,1)$ and

Differentiating $f(x)$ yields

$$
\frac{M(a, b)}{C H(a, b)}=\frac{1}{2 x \operatorname{arcsinh} x} \triangleq f(x) .
$$

$$
f^{\prime}(x)=-\frac{\frac{x}{\sqrt{1+x^{2}}}+\operatorname{arcsinh} x}{2 x^{2} \operatorname{arcsinh}^{2} x} \leq 0
$$

which means that function $f(x)$ is decreasing for $x \in(0,1)$.

It is apparent that

$$
\lim _{x \rightarrow 1^{-}} f(x)=\frac{1}{2 \ln (1+\sqrt{2})} .
$$

The proof of Theorem 2 is thus complete.

Theorem 3. The double inequality

$$
\alpha Q(a, b)+(1-\alpha) M(a, b)<\bar{C}(a, b)<\beta Q(a, b)+(1-\beta) M(a, b)
$$

holds for all $a, b>0$ with $a \neq b$ if and only if $\alpha \leq \frac{1}{2}$ and

$$
\beta \geq \frac{3-4 \ln (1+\sqrt{2})}{3[1-\sqrt{2} \ln (1+\sqrt{2})]}=0.7107 \ldots
$$

Proof. It is sufficient to show

$$
\alpha<\frac{\bar{C}(a, b)-M(a, b)}{Q(a, b)-M(a, b)}<\beta .
$$

Without loss of generality, we assume that $a>b>0$. Let $x=\frac{a}{b}$. Then $x>1$ and

$$
\frac{\bar{C}(a, b)-M(a, b)}{Q(a, b)-M(a, b)}=\frac{\frac{2\left(x^{2}+x+1\right)}{3(x+1)}-\frac{x-1}{2 \operatorname{arcsinh} \frac{x-1}{x+1}}}{\sqrt{\frac{x^{2}+1}{2}}-\frac{x-1}{2 \operatorname{arcsinh} \frac{x-1}{x+1}}} .
$$


Let $t=\frac{x-1}{x+1}$. Then $t \in(0,1)$ and

$$
\frac{\bar{C}(a, b)-M(a, b)}{Q(a, b)-M(a, b)}=\frac{\frac{t^{2}}{3}+1-\frac{t}{\operatorname{arcsinh} t}}{\sqrt{1+t^{2}}-\frac{t}{\operatorname{arcsinh} t}} .
$$

Let $t=\sinh \theta$ for $\theta \in(0, \ln (1+\sqrt{2}))$. Then

$$
\frac{\bar{C}(a, b)-M(a, b)}{Q(a, b)-M(a, b)}=\frac{\frac{\sinh ^{2} \theta}{3}+1-\frac{\sinh \theta}{\theta}}{\cosh \theta-\frac{\sinh \theta}{\theta}} .
$$

By Lemma 3, we obtain Theorem 3.

Theorem 4. The double inequality

$$
\alpha C(a, b)+(1-\alpha) M(a, b)<Q(a, b)<\beta C(a, b)+(1-\beta) M(a, b)
$$

holds for all $a, b>0$ with $a \neq b$ if and only if

$$
\alpha \leq \frac{\sqrt{2} \ln (1+\sqrt{2})-1}{2 \ln (1+\sqrt{2})-1}=0.3231 \ldots \text { and } \beta \geq \frac{2}{5} .
$$

Proof. The double inequalities (4.3) is the same as

$$
\alpha<\frac{Q(a, b)-M(a, b)}{C(a, b)-M(a, b)}<\beta .
$$

Without loss of generality, we assume that $a>b>0$. Let $x=\frac{a}{b}$. Then $x>1$ and

$$
\frac{Q(a, b)-M(a, b)}{C(a, b)-M(a, b)}=\frac{\sqrt{\frac{x^{2}+1}{2}}-\frac{x-1}{2 \operatorname{arcsinh} \frac{x-1}{x+1}}}{\frac{x^{2}+1}{x+1}-\frac{x-1}{2 \operatorname{arcsinh} \frac{x-1}{x+1}}} .
$$

Let $t=\frac{x-1}{x+1}$. Then $t \in(0,1)$ and

$$
\frac{Q(a, b)-M(a, b)}{C(a, b)-M(a, b)}=\frac{\sqrt{1+t^{2}}-\frac{t}{\operatorname{arcsinh} t}}{1+t^{2}-\frac{t}{\operatorname{arcsinh} t}} .
$$

Let $t=\sinh \theta$ for $\theta \in(0, \ln (1+\sqrt{2}))$. Then

$$
\frac{Q(a, b)-M(a, b)}{C(a, b)-M(a, b)}=\frac{\cosh \theta-\frac{\sinh \theta}{\theta}}{1+\sinh ^{2} \theta-\frac{\sinh \theta}{\theta}} .
$$

According to Lemma 4, the proof of Theorem 3 is complete.

Remark 1. This paper is a slightly revised version of the preprint [6].

\section{ACKNOWLEDGEMENTS}

The authors thank the anonymous referees for their careful corrections to the original version of this paper. 


\section{REFERENCES}

[1] Y.-M. Chu and B.-Y. Long, "Bounds of the Neuman-Sándor mean using power and identric means," Abstr. Appl. Anal., pp. Art. ID 832 591, 6 pp., 2013.

[2] Y.-M. Chu, B.-Y. Long, W.-M. Gong, and Y.-Q. Song, "Sharp bounds for Seiffert and Neuman-Sándor means in terms of generalized logarithmic means," J. Inequal. Appl., pp. 2013:10, 13 pp., 2013. [Online]. Available: http://dx.doi.org/10.1186/1029-242X-2013-10

[3] W.-D. Jiang and F. Qi, "Sharp bounds in terms of the power of the contra-harmonic mean for Neuman-Sándor mean,” arXiv preprint. [Online]. Available: http://arxiv.org/abs/1301.3554

[4] W.-D. Jiang and F. Qi, "Some sharp inequalities involving Seiffert and other means and their concise proofs," Math. Inequal. Appl., vol. 15, no. 4, pp. 1007-1017, 2012. [Online]. Available: http://dx.doi.org/10.7153/mia-15-86

[5] W.-D. Jiang and F. Qi, "Sharp bounds for Neuman-Sándor's mean in terms of the root-mean-square," Period. Math. Hungar, p. in press, 2014. [Online]. Available: http: //dx.doi.org/10.1007/s10998-014-0057-9

[6] W.-H. Li and F. Qi, "A unified proof of inequalities and some new inequalities involving Neuman-Sándor mean," arXiv preprint. [Online]. Available: http://arxiv.org/abs/1312.3500

[7] W.-H. Li and M.-M. Zheng, "Some inequalities for bounding Toader mean," J. Funct. Spaces Appl., pp. Art. ID 394 194, 5 pp., 2013.

[8] Y.-M. Li, B.-Y. Long, and Y.-M. Chu, "Sharp bounds for the Neuman-Sándor mean in terms of generalized logarithmic mean," J. Math. Inequal., vol. 6, no. 4, pp. 567-577, 2012. [Online]. Available: http://dx.doi.org/10.7153/jmi-06-54

[9] E. Neuman, "Inequalities for the Schwab-Borchardt mean and their applications," J. Math. Inequal., vol. 5, no. 4, pp. 601-609, 2011. [Online]. Available: http://dx.doi.org/10.7153/ jmi-05-52

[10] E. Neuman, “A note on a certain bivariate mean,” J. Math. Inequal., vol. 6, no. 4, pp. 637-643, 2012. [Online]. Available: http://dx.doi.org/10.7153/jmi-06-62

[11] E. Neuman and J. Sándor, "On the Schwab-Borchardt mean," Math. Pannon., vol. 14, no. 2, pp. 253-266, 2003.

[12] E. Neuman and J. Sándor, "On the Schwab-Borchardt mean. II," Math. Pannon., vol. 17, no. 1, pp. 49-59, 2006.

[13] E. Neuman and J. Sándor, "Companion inequalities for certain bivariate means," Appl. Anal. Discrete Math., vol. 3, no. 1, pp. 46-51, 2009. [Online]. Available: http: //dx.doi.org/10.2298/AADM0901046N

[14] X.-H. Shen and Y.-M. Chu, "Bounds improvement for Neuman-Sándor mean using arithmetic, quadratic and contraharmonic means," Int. Math. Forum, vol. 8, no. 29-32, pp. 1477-1485, 2013.

[15] S. Simić and M. Vuorinen, "Landen inequalities for zero-balanced hypergeometric functions," Abstr. Appl. Anal., pp. Art. ID 932061, 11 pp., 2012. [Online]. Available: http: //dx.doi.org/10.1155/2012/932061

[16] H. Sun, X.-H. Shen, T.-H. Zhao, and Y.-M. Chu, "Optimal bounds for the Neuman-Sándor means in terms of geometric and contraharmonic means," Appl. Math. Sci. (Ruse), vol. 7, no. 85-88, pp. 4363-4373, 2013. [Online]. Available: http://dx.doi.org/10.12988/ams.2013.35247

[17] H. Sun, T. Zhao, Y. Chu, and B. Liu, "A note on the Neuman-Sándor mean," J. Math. Inequal., vol. 8, no. 2, pp. 287-297, 2014. [Online]. Available: http://dx.doi.org/10.7153/jmi-08-20

[18] M.-K. Wang, Y.-M. Chu, and B.-Y. Liu, "Sharp inequalities for the Neuman-Sándor mean in terms of arithmetic and contra-harmonic means," Rev. Anal. Numér. Théor. Approx., vol. 42, no. 2, pp. 115-120, 2013. 
[19] T.-H. Zhao and Y.-M. Chu, "A sharp double inequality involving identric, Neuman-Sándor, and quadratic means," Scientia Sinica Mathematica, vol. 43, no. 6, pp. 551-562, 2013. [Online]. Available: http://dx.doi.org/10.1360/012013-128

[20] T.-H. Zhao, Y.-M. Chu, and B.-Y. Liu, "Optimal bounds for Neuman-Sándor mean in terms of the convex combinations of harmonic, geometric, quadratic, and contraharmonic means," Abstr. Appl. Anal., pp. Art. ID 302635,9 pp., 2012.

\section{Authors' addresses}

\section{Feng Qi}

Department of Mathematics, School of Science, Tianjin Polytechnic University, Tianjin City, 300387 , China, College of Mathematics, Inner Mongolia University for Nationalities, Tongliao City, Inner Mongolia Autonomous Region, 028043, China, Institute of Mathematics, Henan Polytechnic University, Jiaozuo City, Henan Province, 454010, China

E-mail address: qifeng618@gmail.com, qifeng618@gmail.com, qifeng618@hotmail.com, qifeng618@hotmail.com, qifeng618@qq.com, qifeng618@qq.com

URL: qifeng618.wordpress.com

Wen-Hui Li

Department of Mathematics, School of Science, Tianjin Polytechnic University, Tianjin City, 300387, China

E-mail address: wen.hui.lil02@gmail.com, wen.hui.lil02@gmail.com, wen.hui.li@foxmail.com, wen.hui.li@foxmail.com 